\title{
An epidemiological study of petroleum refinery employees
}

\author{
O WONG,${ }^{1}$ R W MORGAN,${ }^{1}$ WJ BAILEY ${ }^{2}$, R E SWENCICKI, ${ }^{2}$ \\ KATHLEEN CLAXTON, ${ }^{1}$ AND LEEKA KHEIFETS ${ }^{1}$
}

From the Environmental Health Associates, Inc, ${ }^{1}$ Oakland, CA 94607, and Chevron Corporation Medical Staff, ${ }^{2}$ San Francisco, CA 94104, USA

ABSTRACT A cohort study of 14179 current and former Chevron USA employees at the Richmond and El Segundo, California, refineries was conducted. The cohort consisted of everyone working at either refinery for a minimum of one year. The observed mortality of the cohort, by cause, was compared with the expected based on the United States mortality rates, standardised for age, race, sex, and calendar time. Analyses by refinery, job category, hire date, duration of employment, and latency were performed. For the entire cohort, mortality from all causes was $72.4 \%$ of that expected, a deficit that was statistically significant. In addition, a significantly lower mortality was found for all forms of cancer combined, digestive cancer, lung cancer, heart disease, non-malignant respiratory disease, diseases of the digestive system, and accidents. Only lymphopoietic cancer showed a pattern of increased risk suggestive of a possible relation to an occupational exposure. The excess appears confined to cancer of lymphatic tissue (not leukaemias) at Richmond, and only among those hired before 1948. A follow up case analysis of the deaths from lymphatic cancer failed to identify a common exposure pattern.

Several epidemiological studies of petroleum refinery workers have been conducted ${ }^{1-10}$ (Divine BJ et al, unpublished report). Although these studies differed in size and design and covered different periods they showed several cancer sites with significant excesses among individuals exposed to petroleum and its byproducts when compared with the general population or with non-exposed employees. The cancer sites that have been implicated in more than one study include cancers of the respiratory system, stomach, lymphopoietic tissues, and, perhaps, brain. Although some of these studies have certain limitations, either because of their design or because of the particular data source available to the investigators, they have suggested possible health risks among refinery employees. To investigate whether these risks existed among its employees, Chevron undertook a long term mortality study at its Richmond and El Segundo, California, refineries.

Accepted 22 April 1985

\section{Materials and methods}

\section{DATA COLLECTION}

The cohort consisted of all individuals who had completed one year of work by 31 December 1980 and $\frac{\Phi}{3}$ who had worked for at least one day between 1 Jan-:uary 1950 and 31 December 1980 at the Chevron 3 . refineries in Richmond and El Segundo, California. The cohort was constructed by using employment 3 records at the two refineries, corporate offices, and $\mathrm{O}$ other Chevron record storage facilities.

For efficiency, only the first and last jobs (job titles,,$\frac{D}{2}$ locations, and dates) were coded. By not coding the entire work history, it is possible to miss specific jobs $N$ of high risk. Nevertheless, we thought that this would $N$ correctly identify most subcohort members yet offer considerable savings in both time for completion and ${ }^{\omega}$ cost of coding. It was also recognised that should the study generate enough interest in certain causes of death, any further investigation would require eithero a detailed case analysis or, ultimately, a case-control ${ }^{-}$ study (with complete job coding of cases and con- $\frac{0}{0}$ trols) if feasible and warranted by the available data. $\overrightarrow{\mathbb{D}}$ A third, less likely, possibility would be a reanalysis of 
the entire cohort, but after adding complete job coding.

The employment records generally included the following information: name, social security number, sex, race, date of birth, date of hire (or service date), date of separation, and an employment history. Dates of death and death certificates were available for some individuals who had died while employed or after retiring.

\section{COHORT VERIFICATION}

We investigated the possibility of using social security quarterly reports (Form 941) to verify the completeness of the cohort but found that that method was not practical because these reports were not specific to locations within the state. The best data source for verification within the company, but independent of the source of data collection, was employee year end tapes from the centralised personnel/payroll system. These computer tapes are made at the end of each calendar year of all employees who had had some employment or retirement activity in that year. Year end tapes are available from 1962.

For verification purposes, three sets of year end tapes (1965-6, 1970-1, 1977-8) were randomly selected within each of the three six year periods (1962-7, 1968-73), 1974-9). A cohort of 7097 individuals were generated from these tapes. Of these, 279 $(3.8 \%)$ were not included in the cohort (183 from Richmond and 96 from El Segundo). About half these $(<2 \%)$ were found to be eligible for the cohort but were missed in our data collection, either because they were not present in the files at the time of microfilming or were inadvertently omitted from the microfilming. The few potential cohort members missed was not over represented in any particular occupational department or file storage facility and we do not believe, therefore, that any bias in cohort ascertainment would be likely to result.

VITAL STATUS FOLLOW UP

It was necessary to determine whether those workers who had terminated their employment (including terminees, transferred, and retirees) before 31 December 1980 were still living or whether they had died between leaving and the end of the study. Sources used in the vital status follow up of former employees included:

(1) Social Security Administration Vital Status Follow up,

(2) California Automated Mortality Linkage System (University of California, San Francisco), and

(3) the California Department of Motor Vehicles.
DEATH CERTIFICATE ACQUISITION

We retrieved death certificates for 2242 , or $97.82 \%$ of those identified as dead, most of whom had died in California. All death certificates were coded by a trained nosologist according to the 8th revision of the International Classification of Diseases by underlying cause of death. ${ }^{11}$

\section{STATISTICAL PROCEDURE}

The most common summary index for assessing the risk of death in a cohort study is the standardised mortality ratio (SMR). The basic unit of computation is the number of years each employee was followed up (person-years) from the anniversary date (one year of service) or 1 January 1950 (whichever was later) to the end of the study period or the date of death (whichever was earlier). For those "lost to follow up," person-years of observation were counted up to the last date of contact, which was usually the termination date or the last driver's license renewal date. Thereafter, these individuals were taken out of the analysis. This amounts to assuming that the mortality experience of these individuals was similar to that of the rest of the cohort. Each year (or fraction thereof) contributed by an individual worker was classified by age, race, sex, and calendar year, and these personyears of all workers were then summed up by age, race, sex, and calendar year. The United States national age-cause-race-sex-specific mortality rates for five year periods from 1950 to 1980 were applied to those person-years to obtain the number of deaths from a particular cause to be expected from an equal number of person-years of the same race and sex and similar in age and calendar year.

SMRs were computed by expressing the actually observed deaths as percentages of the expected. An SMR higher or lower than 100 indicated an excess or a deficit in mortality. The deviation from 100 was tested to determine whether it was statistically significant at the $5 \%$ and $1 \%$ levels. The actual computation was performed using a standard computer program. $^{12}$

\section{Results}

DESCRIPTIVE STATISTICS

Altogether 14179 individuals who satisfied the cohort

Table 1 Distribution by sex and race of all cohort members at Richmond and El Segundo refineries

\begin{tabular}{lrcr}
\hline Race & Men & Woman & Total \\
\hline White & 10127 & 508 & 10635 \\
Non-white & 718 & 54 & 772 \\
Unknown & 2656 & 116 & 2772 \\
Total & 13501 & 678 & 14179 \\
\hline
\end{tabular}


Table 2 Distribution by year of hire of all cohort members at Richmond and El Segundo refineries

\begin{tabular}{|c|c|c|c|c|c|c|}
\hline \multirow[t]{2}{*}{ Year of hire } & \multicolumn{2}{|l|}{ Richmond } & \multicolumn{2}{|l|}{ El Segundo } & \multicolumn{2}{|c|}{ Both refineries } \\
\hline & Frequency & $\%$ & Frequency & $\%$ & Frequency & $\%$ \\
\hline $\begin{array}{c}<1920 \\
1920-4 \\
1925-9 \\
1930-4 \\
1935-9 \\
1940-4 \\
1945-9 \\
1950-4 \\
1955-9 \\
1960-4 \\
1965-9 \\
1970-4 \\
1975-9 \\
\text { Total }\end{array}$ & $\begin{array}{r}258 \\
229 \\
137 \\
91 \\
356 \\
750 \\
1788 \\
984 \\
946 \\
762 \\
847 \\
861 \\
972 \\
8981\end{array}$ & $\begin{array}{r}2.88 \\
2.55 \\
1.53 \\
1.01 \\
3.96 \\
8.35 \\
19.91 \\
10.96 \\
10.53 \\
8.48 \\
9.43 \\
9.59 \\
10.82 \\
100.00\end{array}$ & $\begin{array}{r}65 \\
133 \\
115 \\
77 \\
167 \\
439 \\
837 \\
631 \\
523 \\
375 \\
517 \\
671 \\
648 \\
5198\end{array}$ & $\begin{array}{r}1.25 \\
2.56 \\
2.21 \\
1.48 \\
3.21 \\
8.45 \\
16.10 \\
12.14 \\
10.06 \\
7.21 \\
9.95 \\
12.91 \\
12.47 \\
100.00\end{array}$ & $\begin{array}{r}323 \\
362 \\
252 \\
168 \\
523 \\
1189 \\
2625 \\
1615 \\
1469 \\
1137 \\
1364 \\
1532 \\
1620 \\
14179\end{array}$ & $\begin{array}{r}2.28 \\
2.55 \\
1 \cdot 78 \\
1 \cdot 18 \\
3.69 \\
8 \cdot 39 \\
18 \cdot 51 \\
11 \cdot 39 \\
10.36 \\
8 \cdot 02 \\
9.62 \\
10.80 \\
11 \cdot 43 \\
100 \cdot 00\end{array}$ \\
\hline
\end{tabular}

criteria were included in the analysis. Table 1 gives their sex and race distribution. Table 2 shows the distribution by year of hire for the two refineries and for the entire cohort. Major hiring at the two refineries occurred in the late $1940 \mathrm{~s}$. Almost $40 \%$ of the cohort were hired before 1950, thus providing a possible latency of up to 30 years for this group.

Table 3 shows the distribution by duration of employment for the entire cohort. Over $47 \%$ of the cohort was employed for 10 years or more and $33.5 \%$ were employed for less than five years. The average duration of employment for the entire cohort was about 14 years.

Table 4 shows the vital status of the cohort on 31 December 1980. At the end of the study period, $11177(78.83 \%)$ were still alive. A total of 2292 individuals had died during the study period and death certificates were obtained for all except $50(2 \cdot 18 \%$ of all deaths). At the end of the study, the vital status of 710 individuals $(5 \cdot 01 \%)$ remained unknown.

\section{CAUSE SPECIFIC MORTALITY ANALYSIS}

The race of 2772 individuals was not available from their employment records. There were, however, few known non-whites (772) among the Chevron employees at the two refineries included in this analysis. In order not to bias any race specific analysis, race infor-

Table 3 Distribution by duration of employment of all cohort members at Richmond and El Segundo refineries

\begin{tabular}{lcr}
\hline Employment duration (years) & Frequency & $\%$ \\
\hline$<5$ & 4753 & $33 \cdot 52$ \\
$5-9$ & 2752 & $19 \cdot 41$ \\
$10-14$ & 1417 & $9 \cdot 99$ \\
$15-19$ & 823 & $5 \cdot 80$ \\
$20-24$ & 837 & $5 \cdot 90$ \\
$25-29$ & 1194 & $8 \cdot 42$ \\
$30-34$ & 1292 & $9 \cdot 11$ \\
$35-39$ & 724 & $5 \cdot 11$ \\
$\geqslant 40$ & 387 & $2 \cdot 73$ \\
Total & 14179 & $100 \cdot 00$ \\
\hline
\end{tabular}

mation from death certificates was not used and for the analysis, those individuals with unknown race (2772) were assumed to be white.

In the study 2292 deaths were identified. Death certificates were obtained for 2242 . The 50 deaths without death certificates, but with dates of death, were coded as cause unknown. These 50 deaths were included in the calculation of overall SMRs but not in any cause specific SMRs.

ANALYSIS OF TOTAL COHORT AND BY REFINERY Table 5 shows the observed deaths and SMRs by cause for each refinery and the total cohort. For both refineries, the total mortality was significantly low (SMR = 74.1 at Richmond, 69.2 at El Segundo; 72.4 for both) by comparison with the United States national mortality experience. For most causes of death, the SMRs at the two refineries were similar, and our discussion will focus on the total cohort. Mortality from cancer of all sites combined for the total cohort was also significantly lower than the

Table 4 Distribution by vital status (on 31 December 1980) of all cohort members

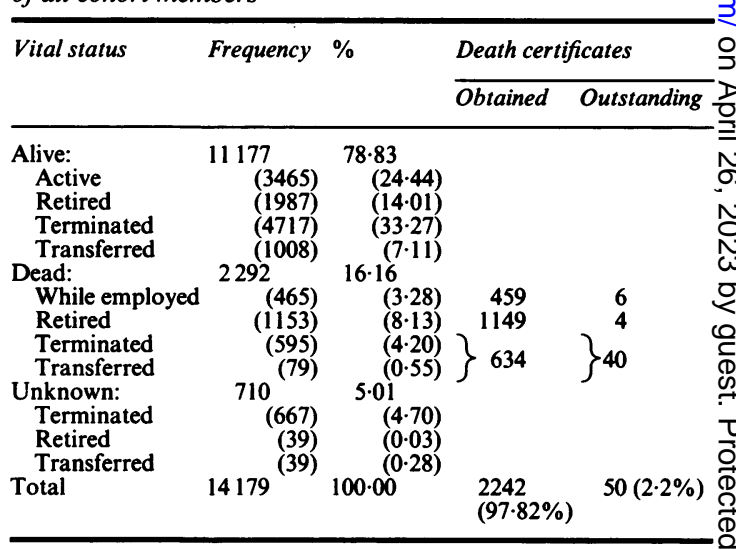


Table 5 Observed deaths and SMRs by cause for total cohort at Richmond and El Segundo refineries

\begin{tabular}{|c|c|c|c|c|c|c|}
\hline \multirow[t]{2}{*}{ Cause of death (8th ICDA) } & \multicolumn{2}{|l|}{ Total } & \multicolumn{2}{|c|}{ Richmond } & \multicolumn{2}{|c|}{ El Segundo } \\
\hline & Obs & $S M R$ & $O b s$ & $S M R$ & Obs & $S M R$ \\
\hline $\begin{array}{l}\text { All causes } \\
\text { Infective \& parasitic diseases (000-139) } \\
\text { All cancers (140-209) } \\
\text { Cancer of buccal cavity \& pharynx (140-149) } \\
\text { Cancer of digestive system (150-159) } \\
\text { Cancer of oesophagus (150) } \\
\text { Cancer of stomach (151) } \\
\text { Cancer of large intestine (153) } \\
\text { Cancer of rectum (154) } \\
\text { Cancer of liver (155-156) } \\
\text { Cancer of pancreas (157) } \\
\text { Cancer of respiratory system (160-163) } \\
\text { Cancer of larynx (161) } \\
\text { Cancer of lung (162-163) } \\
\text { Cancer of skin (172-173) } \\
\text { Cancer of breast (174) } \\
\text { Cancer of prostate (185) } \\
\text { Cancer of testis (186-187) } \\
\text { Cancer of bladder (188) } \\
\text { Cancer of kidney (189) } \\
\text { Cancer of brain and CNS (191-192) } \\
\text { Lymphatic \& haematopoietic cancer (200-209) } \\
\text { Lymphosarcoma \& reticulosarcoma (200) } \\
\text { Hodgkin's disease (201) } \\
\text { Leukaemia \& aleukaemia (204-207) } \\
\text { Other lymphatic tissue cancers (202, 203, 208) } \\
\text { Benign neoplasms (210-239) } \\
\text { Diabetes mellitus (250) } \\
\text { Diseases of blood (280-289) } \\
\text { Diseases of nervous system (320-389) } \\
\text { Diseases of circulatory system (390-458) } \\
\text { Chronic rheumatic heart disease (393-398) } \\
\text { Arteriosclerotic heart disease (410-413) } \\
\text { Vascular lesions of CNS (430-438) } \\
\text { Non-malignant respiratory disease (460-519) } \\
\text { Pneumonia (480-486) } \\
\text { Emphysema (492) } \\
\text { Diseases of digestive system (520-577) } \\
\text { Cirrhosis of liver (551) } \\
\text { Diseases of genitourinary system (580-629) } \\
\text { Senility \& ill-defined conditions (780-799) } \\
\text { Accidents, poisonings, \& violence (E800-E998) } \\
\text { Accidents ( } 800-949) \\
\text { Motor vehicle accidents (810-827) } \\
\text { Suicide (950-959) }\end{array}$ & $\begin{array}{r}2292 \\
14 \\
462 \\
9 \\
125 \\
12 \\
26 \\
40 \\
18 \\
6 \\
20 \\
135 \\
9 \\
124 \\
10 \\
3 \\
34 \\
2 \\
12 \\
13 \\
22 \\
64 \\
17 \\
4 \\
22 \\
20 \\
5 \\
17 \\
6 \\
12 \\
1228 \\
19 \\
924 \\
158 \\
105 \\
35 \\
26 \\
97 \\
60 \\
22 \\
10 \\
243 \\
146 \\
74 \\
88\end{array}$ & $\begin{array}{c}72 \cdot 4 \dagger \\
37 \cdot 7 \dagger \\
75 \cdot 7 \dagger \\
47 \cdot 0^{*} \\
71 \cdot 6 \dagger \\
83 \cdot 5 \\
76 \cdot 2 \\
71 \cdot 4^{*} \\
89 \cdot 1 \\
49 \cdot 0 \\
59 \cdot 2^{*} \\
68 \cdot 7 \dagger \\
99 \cdot 6 \\
66 \cdot 9 \dagger \\
92 \cdot 5 \\
101 \cdot 8 \\
78 \cdot 4 \\
53 \cdot 0 \\
62 \cdot 4 \\
87 \cdot 7 \\
125 \cdot 9 \\
105 \cdot 0 \\
126 \cdot 6 \\
52 \cdot 1 \\
88 \cdot 3 \\
141 \cdot 6 \\
61 \cdot 5 \\
37 \cdot 1 \dagger \\
85 \cdot 2 \\
47 \cdot 6 \dagger \\
73 \cdot 6 \dagger \\
61 \cdot 1 * \\
79 \cdot 3 \dagger \\
66 \cdot 6 \dagger \\
55 \cdot 7 \dagger \\
49 \cdot 5 \dagger \\
53 \cdot 4 \dagger \\
64 \cdot 7 \dagger \\
79 \cdot 0 \\
49 \cdot 8 \dagger \\
27 \cdot 2 \dagger \\
79 \cdot 2 \dagger \\
71 \cdot 2 \dagger \\
77 \cdot 0 * \\
127 \cdot 6^{*}\end{array}$ & $\begin{array}{r}1507 \\
5 \\
302 \\
7 \\
85 \\
8 \\
21 \\
27 \\
13 \\
2 \\
13 \\
82 \\
6 \\
74 \\
7 \\
2 \\
23 \\
2 \\
10 \\
9 \\
15 \\
46 \\
13 \\
3 \\
14 \\
15 \\
2 \\
12 \\
4 \\
10 \\
801 \\
8 \\
605 \\
105 \\
71 \\
21 \\
17 \\
68 \\
41 \\
15 \\
7 \\
162 \\
99 \\
51 \\
56\end{array}$ & $\begin{array}{c}74 \cdot 1 \dagger \\
20 \cdot 7 \dagger \\
77 \cdot 5 \dagger \\
57 \cdot 2 \\
76 \cdot 0 \dagger \\
86 \cdot 4 \\
95 \cdot 3 \\
75 \cdot 7 \\
100 \cdot 5 \\
25 \cdot 4 \\
60 \cdot 3 \\
65 \cdot 6 \dagger \\
104 \cdot 0 \\
62 \cdot 8 \dagger \\
101 \cdot 2 \\
127 \cdot 2 \\
82.6 \\
81 \cdot 1 \\
81 \cdot 6 \\
95 \cdot 2 \\
134 \cdot 5 \\
118 \cdot 0 \\
151 \cdot 3 \\
60 \cdot 4 \\
87 \cdot 8 \\
167 \cdot 0 \\
38 \cdot 4 \\
40 \cdot 8 \dagger \\
88 \cdot 2 \\
61 \cdot 8 \\
74.9 \dagger \\
40 \cdot 1 \dagger \\
81 \cdot 2 \dagger \\
68 \cdot 5 \dagger \\
59 \cdot 0 \dagger \\
46 \cdot 1 \dagger \\
55 \cdot 1 \dagger \\
70 \cdot 7 \dagger \\
84 \cdot 4 \\
52 \cdot 3 \dagger \\
29 \cdot 3 \dagger \\
80.9 \dagger \\
73 \cdot 9 \dagger \\
80 \cdot 8 \\
126 \cdot 2\end{array}$ & $\begin{array}{r}785 \\
9 \\
160 \\
2 \\
40 \\
4 \\
5 \\
13 \\
5 \\
4 \\
7 \\
53 \\
3 \\
50 \\
3 \\
1 \\
11 \\
0 \\
2 \\
4 \\
7 \\
18 \\
4 \\
1 \\
8 \\
5 \\
3 \\
5 \\
2 \\
2 \\
427 \\
11 \\
319 \\
53 \\
34 \\
14 \\
9 \\
29 \\
19 \\
7 \\
23 \\
32\end{array}$ & $\begin{array}{c}69 \cdot 2 \dagger \\
69 \cdot 3 \\
72 \cdot 3 \dagger \\
29 \cdot 0 \\
63 \cdot 7 \dagger \\
78 \cdot 1 \\
41 \cdot 4^{*} \\
64 \cdot 0 \\
68 \cdot 8 \\
91 \cdot 7 \\
57 \cdot 3 \\
74 \cdot 1 \\
91 \cdot 9 \\
74 \cdot 0^{*} \\
77 \cdot 1 \\
72 \cdot 8 \\
70 \cdot 9 \\
28.7 \\
74 \cdot 5 \\
110 \cdot 7 \\
81 \cdot 9 \\
82 \cdot 8 \\
36 \cdot 8 \\
89 \cdot 1 \\
97 \cdot 3 \\
102 \cdot 5 \\
30 \cdot 5 \dagger \\
79 \cdot 7 \\
22 \cdot 2^{*} \\
71 \cdot 4 \dagger \\
98 \cdot 4 \\
75 \cdot 9 \dagger \\
63 \cdot 0 \dagger \\
49 \cdot 8 \dagger \\
55 \cdot 5^{*} \\
50 \cdot 5^{*} \\
54 \cdot 0 \dagger \\
69 \cdot 5 \\
45 \cdot 3^{*} \\
23 \cdot 2 \dagger \\
76 \cdot 1^{*} \\
66 \cdot 1 \dagger \\
69 \cdot 8 \\
130 \cdot 3\end{array}$ \\
\hline
\end{tabular}

* Significant at 0.05 .

+Significant at $0 \cdot 01$.

United States experience. The deficit came primarily from the following sites: cancer of buccal cavity and pharynx (SMR $=47.0, \mathrm{p}<0.05)$, digestive system $(\mathrm{SMR}=71.6, \mathrm{p}<0.01)$, and lung $(\mathrm{SMR}=66.9$, $p<0.01)$. Non-significant deficits were also observed for several other sites, including cancer of the prostate and bladder, Hodgkin's disease, and leukaemia.

Slight, but non-significant, increases in mortality were found for cancer of the brain (SMR $=125.9)$, lymphosarcoma and reticulosarcoma (SMR = 126.6), and other lymphatic tissue cancers $(\mathrm{SMR}=141 \cdot 6)$. The excesses came primarily from the Richmond refinery, which experienced an SMR of 134.4 for brain cancer, 151.3 for lymphosarcoma and reticulosarcoma, and 167.0 for other lymphatic tissue cancers; none of these SMRs was statistically significant.

Mortality from cancer of the larynx $(\mathrm{SMR}=99 \cdot 6)$, skin $(S M R=92.5)$, breast $(S M R=101.8)$, and kidney $(\mathrm{SMR}=87.7)$ for the total cohort was similar to that expected.

Both benign neoplasms and diseases of the blood showed a non-significant deficit (SMR $=61 \cdot 5$ and $85 \cdot 2$, respectively). Statistically significant mortality deficits were observed, however, for many nonmalignant causes: diseases of the nervous system $(\mathrm{SMR}=47.6, \mathrm{p}<0.01)$ and circulatory system (SMR $=73.6, \mathrm{p}<0.01)$, non-malignant respiratory diseases $(\mathrm{SMR}=55.7, \mathrm{p}<0.01)$, diseases of the digestive system $(\mathrm{SMR}=64.7, \mathrm{p}<0.01)$, and genito- 
Table 6 Observed deaths by cause and SMRs for all cohort members in laboratory, maintenance, and operating locations at Richmond and El Segundo refineries

\begin{tabular}{|c|c|c|c|c|c|c|}
\hline \multirow[t]{2}{*}{ Cause of death (8th ICDA) } & \multicolumn{2}{|c|}{ Laboratory } & \multicolumn{2}{|c|}{ Maintenance } & \multicolumn{2}{|c|}{ Operating } \\
\hline & $\overline{O b s}$ & $S M R$ & $\overline{O b s}$ & $S M R$ & $\overline{O b s}$ & SMR \\
\hline $\begin{array}{l}\text { All causes } \\
\text { Infective \& parasitic diseases (000-139) } \\
\text { All cancers (140-209) } \\
\text { Cancer of buccal cavity \& pharynx (140-149) } \\
\text { Cancer of digestive system (150-159) } \\
\text { Cancer of oesophagus (150) } \\
\text { Cancer of stomach (151) } \\
\text { Cancer of large intestine (153) } \\
\text { Cancer of rectum (154) } \\
\text { Cancer of liver (155-156) } \\
\text { Cancer of pancreas (157) } \\
\text { Cancer of respiratory system (160-163) } \\
\text { Cancer of larynx (161) } \\
\text { Cancer of lung (162-163) } \\
\text { Cancer of skin (172-173) } \\
\text { Cancer of breast (174) } \\
\text { Cancer of prostate (185) } \\
\text { Cancer of testis (186-187) } \\
\text { Cancer of bladder (188) } \\
\text { Cancer of kidney (189) } \\
\text { Cancer of brain and CNS (191-192) } \\
\text { Lymphatic \& haematopoietic cancer (200-209) } \\
\text { Lymphosarcoma \& reticulosarcoma (200) } \\
\text { Hodgkin's disease (201) } \\
\text { Leukaemia \& aleukaemia (204-207) } \\
\text { Other lymphatic tissue cancers (202, 203, 208) } \\
\text { Benign neoplasms (210-239) } \\
\text { Diabetes mellitus (250) } \\
\text { Diseases of blood (280-289) } \\
\text { Diseases of nervous system (320-389) } \\
\text { Diseases of circulatory system (390-458) } \\
\text { Chronic rheumatic heart disease (393-398) } \\
\text { Arteriosclerotic heart disease (410-413) } \\
\text { Vascular lesions of CNS (430-438) } \\
\text { Non-malignant respiratory disease (460-519) } \\
\text { Pneumonia (480-486) } \\
\text { Emphysema (492) } \\
\text { Diseases of digestive system (520-577) } \\
\text { Cirrhosis of liver (551) } \\
\text { Diseases of genitourinary system (580-629) } \\
\text { Senility \& ill-defined conditions (780-799) } \\
\text { Accidents, poisonings, \& violence (E800-E998) } \\
\text { Accidents ( } 800-949) \\
\text { Motor vehicle accidents (810-827) } \\
\text { Suicide (950-959) }\end{array}$ & $\begin{array}{r}134 \\
0 \\
29 \\
0 \\
7 \\
0 \\
0 \\
2 \\
4 \\
0 \\
1 \\
3 \\
0 \\
3 \\
2 \\
0 \\
3 \\
0 \\
1 \\
2 \\
1 \\
6 \\
2 \\
0 \\
1 \\
3 \\
0 \\
2 \\
0 \\
1 \\
71 \\
0 \\
57 \\
6 \\
5 \\
1 \\
2 \\
4 \\
3 \\
1 \\
1 \\
17 \\
7 \\
1 \\
9\end{array}$ & 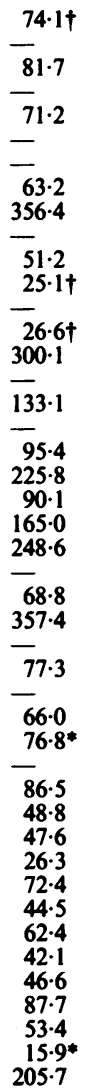 & $\begin{array}{r}1329 \\
12 \\
266 \\
4 \\
70 \\
7 \\
15 \\
17 \\
13 \\
5 \\
12 \\
80 \\
7 \\
72 \\
7 \\
1 \\
20 \\
2 \\
7 \\
7 \\
14 \\
36 \\
10 \\
2 \\
13 \\
11 \\
2 \\
7 \\
3 \\
6 \\
719 \\
9 \\
541 \\
93 \\
61 \\
21 \\
12 \\
58 \\
34 \\
11 \\
90 \\
41 \\
44\end{array}$ & $\begin{array}{c}72.2 \dagger \\
56.0 \\
75.0 \dagger \\
35.8 \\
68.8 \dagger \\
82.6 \\
75.2 \\
52.4 \dagger \\
110.6 \\
70.7 \\
60.8 \\
69.1 \dagger \\
131.6 \\
65.9 \dagger \\
112.5 \\
515.0 \\
76.9 \\
93.1 \\
61.7 \\
80.9 \\
139.9 \\
102.3 \\
129.2 \\
45.9 \\
90.2 \\
133.7 \\
43.1 \\
26.5 \dagger \\
74.3 \\
41.6 * \\
73.6 \dagger \\
51.0 \\
79.1 \dagger \\
67.0 \dagger \\
55.0 \dagger \\
50.6 \dagger \\
41.6 \dagger \\
67.0 \dagger \\
77.7 \\
42.8 \dagger \\
28.1 \dagger \\
80.5 \dagger \\
78.0 \\
76.7 \\
113.0\end{array}$ & $\begin{array}{r}817 \\
2 \\
163 \\
6 \\
36 \\
0 \\
10 \\
13 \\
5 \\
2 \\
4 \\
54 \\
4 \\
50 \\
2 \\
0 \\
7 \\
1 \\
6 \\
3 \\
7 \\
28 \\
7 \\
3 \\
10 \\
7 \\
3 \\
4 \\
5 \\
5 \\
421 \\
2 \\
46 \\
39 \\
15 \\
9 \\
38 \\
26 \\
5 \\
2 \\
33 \\
\end{array}$ & 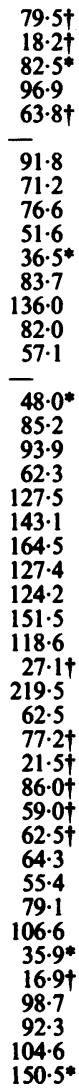 \\
\hline
\end{tabular}

*Significant at 0.05 .

†Significant at 0.01.

urinary system $(\mathrm{SMR}=49.8, \mathrm{p}<0.01)$, senility and ill defined conditions $(\mathrm{SMR}=27 \cdot 2, \mathrm{p}<0.01)$, and accidents (SMR $=71 \cdot 2, \mathrm{p}<0.01)$.

Among all the causes of death examined, mortality from only one cause, suicide, was significantly raised $(\mathrm{SMR}=127.6, \mathrm{p}<0.05)$. Both refineries showed an equal amount of increased mortality from suicide.

\section{ANALYSIS OF WORK LOCATION}

As mentioned earlier, the locations as well as the job titles for the first and last jobs were coded. The locations were classified into several broad categories. Three of the major categories were:

Operating - Light oil, lube oil, thermal cracking, catalytic cracking, chemical and packaging.

Maintenance-Field maintenance, shops, equipment inspection, labour, and engineering.

Laboratory-Analytical laboratories, chemical lab-o oratories, physical laboratories, inspection, and tech: $N$ nical.

In this analysis cohort members were grouped by $O$ location (either the first or last job). Some of the members were in two groups. This analysis repre- $\frac{C}{D}$ sented an attempt to identify any association between $\stackrel{\mathcal{D}}{+}$ general work locations within the refineries and mor- 0 tality. Detailed analysis (such as by duration at a certain location, combination of location, and job title) was deemed inappropriate, since only the first and last 
Table 7 Observed deaths by cause and SMRs for the total cohort at Richmond and El Segundo refineries by latency

\begin{tabular}{|c|c|c|c|c|c|c|}
\hline \multirow[t]{3}{*}{ Cause of death (8th ICDA) } & \multicolumn{6}{|c|}{ Latency } \\
\hline & \multicolumn{2}{|c|}{$<10$ years } & \multicolumn{2}{|c|}{$10-19$ years } & \multicolumn{2}{|c|}{$\geqslant 20$ years } \\
\hline & Obs & $S M R$ & $\overline{O b s}$ & $S M R$ & $\overline{O b s}$ & $S M R$ \\
\hline $\begin{array}{l}\text { All causes } \\
\text { Infective \& parasitic diseases (000-139) } \\
\text { All cancers (140-209) } \\
\text { Cancer of buccal cavity \& pharynx (140-149) } \\
\text { Cancer of digestive system (150-159) } \\
\text { Cancer of oesophagus (150) } \\
\text { Cancer of stomach (151) } \\
\text { Cancer of large intestine (153) } \\
\text { Cancer of rectum (154) } \\
\text { Cancer of liver (155-156) } \\
\text { Cancer of pancreas (157) } \\
\text { Cancer of respiratory system (160-163) } \\
\text { Cancer of larynx (161) } \\
\text { Cancer of lung (162-163) } \\
\text { Cancer of skin (172-173) } \\
\text { Cancer of breast (174) } \\
\text { Cancer of prostate (185) } \\
\text { Cancer of testis (186-187) } \\
\text { Cancer of bladder (188) } \\
\text { Cancer of kidney (189) } \\
\text { Cancer of brain and CNS (191-192) } \\
\text { Lymphatic \& haematopoietic cancer (200-209) } \\
\text { Lymphosarcoma \& reticulosarcoma (200) } \\
\text { Hodgkin's disease (201) } \\
\text { Leukaemia \& aleukaemia (204-207) } \\
\text { Other lymphatic tissue cancers }(202,203,208) \\
\text { Benign neoplasms (210-239) } \\
\text { Diabetes mellitus (250) } \\
\text { Diseases of blood (280-289) } \\
\text { Diseases of nervous system (320-389) } \\
\text { Diseases of circulatory system (390-458) } \\
\text { Chronic rheumatic heart disease (393-398) } \\
\text { Arteriosclerotic heart disease (410-413) } \\
\text { Vascular lesions of CNS (430-438) } \\
\text { Non-malignant respiratory disease (460-519) } \\
\text { Pneumonia (480-486) } \\
\text { Emphysema (492) } \\
\text { Diseases of digestive system (520-577) } \\
\text { Cirrhosis of liver (551) } \\
\text { Diseases of genitourinary system (580-629) } \\
\text { Senility \& ill-defined conditions (780-799) } \\
\text { Accidents, poisonings, \& violence (E800-E998) } \\
\text { Accidents (800-949) } \\
\text { Motor vehicle accidents (810-827) } \\
\text { Suicide ( } 950-959)\end{array}$ & $\begin{array}{r}169 \\
2 \\
21 \\
0 \\
2 \\
0 \\
0 \\
2 \\
0 \\
0 \\
0 \\
8 \\
0 \\
8 \\
1 \\
0 \\
0 \\
0 \\
0 \\
2 \\
2 \\
2 \\
0 \\
0 \\
1 \\
1 \\
1 \\
0 \\
0 \\
1 \\
43 \\
2 \\
33 \\
5 \\
2 \\
1 \\
0 \\
8 \\
4 \\
4 \\
7 \\
79 \\
32 \\
18\end{array}$ & 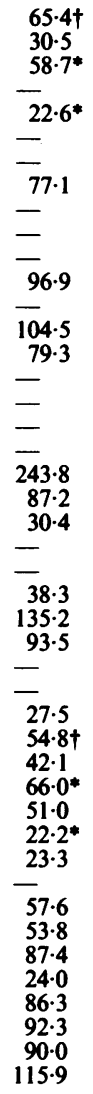 & $\begin{array}{r}388 \\
2 \\
72 \\
1 \\
14 \\
1 \\
5 \\
2 \\
4 \\
0 \\
2 \\
25 \\
2 \\
23 \\
2 \\
1 \\
0 \\
1 \\
0 \\
3 \\
7 \\
12 \\
5 \\
1 \\
1 \\
5 \\
0 \\
0 \\
1 \\
1 \\
186 \\
5 \\
152 \\
12 \\
12 \\
2 \\
7 \\
20 \\
15 \\
3 \\
1 \\
71 \\
41 \\
19 \\
30\end{array}$ & 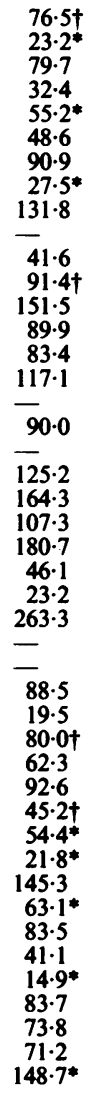 & $\begin{array}{r}1735 \\
10 \\
369 \\
8 \\
109 \\
11 \\
21 \\
36 \\
14 \\
6 \\
18 \\
102 \\
7 \\
93 \\
7 \\
2 \\
34 \\
1 \\
12 \\
8 \\
13 \\
50 \\
12 \\
3 \\
20 \\
14 \\
4 \\
17 \\
5 \\
10 \\
999 \\
12 \\
739 \\
141 \\
91 \\
32 \\
19 \\
69 \\
41 \\
15 \\
8 \\
93 \\
47 \\
23 \\
40\end{array}$ & 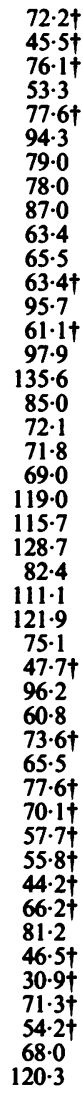 \\
\hline
\end{tabular}

*Significant at 0.05 .

†Significant at 0.01.

jobs were coded.

Table 6 shows the mortality of the subcohorts of employees in laboratory, maintenance, and operating locations. Among the 844 employees in the laboratory locations, the overall SMR for the two refineries combined was 74.1 which was significant at the 0.01 level. In addition, three causes of death showed a significant deficit: lung cancer (SMR $=26 \cdot 6$ ), diseases of the circulatory system $(\mathrm{SMR}=76.8)$, and motor vehicle accidents (SMR = 15.9). None of the calculated SMRs was significantly raised.

About half the cohort members (7838) were classified as having worked in maintenance locations and their overall mortality $(S M R=72 \cdot 2)$ was significantly lower than expected (table 6). Significant mortality deficits were found for all cancers (SMR = 75.0), digestive cancer $(\mathrm{SMR}=68 \cdot 8)$, lung cancer ( $S M R=65.9)$, diseases of the circulatory system $(S M R=73 \cdot 6)$, non-malignant respiratory disease $(S M R=55 \cdot 0)$, and accidents $(S M R=78 \cdot 0)$. Slight, but non-significant, mortality excesses were detected for cancer of larynx, cancer of brain, lymphosarcoma and reticulosarcoma, and other lymphatic tissue cancers.

The 5510 cohort members classified as operating (manufacturing) personnel showed significant deficits 
Table 8 Observed deaths by cause and SMRs for the total cohort at Richmond and El Segundo refineries by duration of employment

\begin{tabular}{|c|c|c|c|c|c|c|}
\hline \multirow[t]{3}{*}{ Cause of death (8th ICDA) } & \multicolumn{6}{|c|}{ Duration of employment } \\
\hline & \multicolumn{2}{|c|}{$<5$ years } & \multicolumn{2}{|c|}{$5-14$ years } & \multicolumn{2}{|c|}{$\geqslant 15$ years } \\
\hline & $\overline{O b s}$ & $S M R$ & $\overline{O b s}$ & $S M R$ & $\overline{O b s}$ & $\overline{S M R}$ \\
\hline 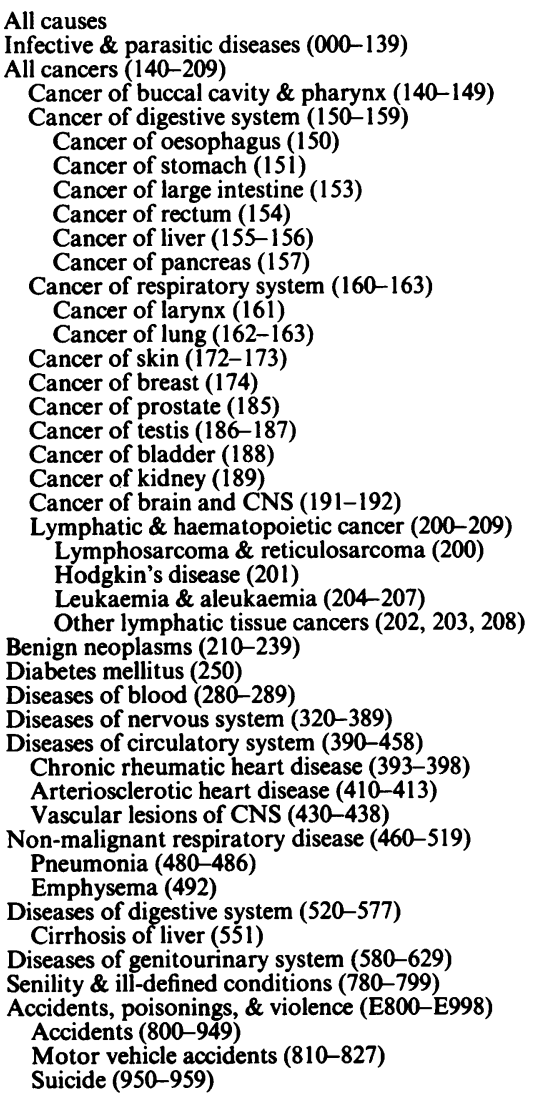 & $\begin{array}{r}262 \\
2 \\
47 \\
0 \\
7 \\
0 \\
0 \\
4 \\
1 \\
0 \\
2 \\
21 \\
0 \\
21 \\
1 \\
0 \\
0 \\
1 \\
0 \\
4 \\
3 \\
3 \\
0 \\
0 \\
0 \\
3 \\
1 \\
0 \\
0 \\
1 \\
84 \\
2 \\
64 \\
13 \\
6 \\
1 \\
2 \\
17 \\
14 \\
3 \\
1 \\
79 \\
56 \\
30 \\
20\end{array}$ & 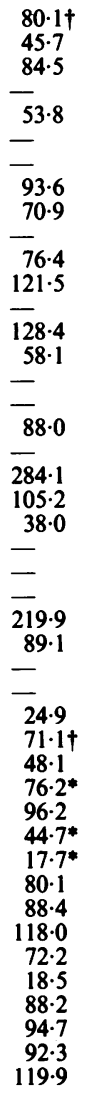 & $\begin{array}{r}525 \\
1 \\
89 \\
1 \\
17 \\
3 \\
2 \\
5 \\
4 \\
0 \\
2 \\
34 \\
1 \\
33 \\
4 \\
1 \\
4 \\
0 \\
0 \\
3 \\
8 \\
11 \\
3 \\
1 \\
2 \\
5 \\
1 \\
3 \\
2 \\
2 \\
256 \\
4 \\
202 \\
23 \\
25 \\
8 \\
6 \\
27 \\
19 \\
4 \\
55 \\
83 \\
52 \\
24 \\
27\end{array}$ & 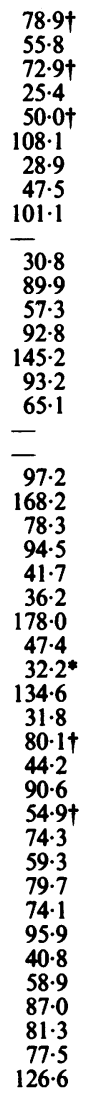 & $\begin{array}{r}1505 \\
6 \\
326 \\
8 \\
101 \\
9 \\
24 \\
31 \\
13 \\
6 \\
16 \\
80 \\
8 \\
70 \\
5 \\
2 \\
30 \\
1 \\
12 \\
6 \\
11 \\
50 \\
14 \\
3 \\
20 \\
12 \\
3 \\
14 \\
4 \\
9 \\
888 \\
13 \\
658 \\
122 \\
74 \\
26 \\
18 \\
53 \\
27 \\
15 \\
4 \\
81 \\
38 \\
20 \\
41\end{array}$ & 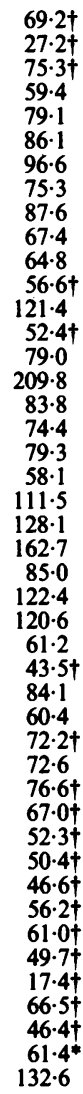 \\
\hline
\end{tabular}

*Significant at 0.05

†Significant at 0.01.

for all causes $(\mathrm{SMR}=79 \cdot 5)$, all cancers (SMR $=82 \cdot 5)$, digestive cancer $(\mathrm{SMR}=63 \cdot 8)$, cancer of prostate $(\mathrm{SMR}=48 \cdot 0)$, diseases of the circulatory system $(\mathrm{SMR}=\mathbf{7 7 \cdot 2})$, and non-malignant respiratory disease $(S M R=62 \cdot 5)$. Non-significant excesses were found for cancer of larynx, lymphosarcoma and reticulosarcoma, other lymphatic tissue cancers, and diseases of the blood. A significant excess was detected for suicide (SMR $=150.5, \mathrm{p}<0.05)$.

ANALYSIS BY LATENCY

Long latent periods are usually required for chronic diseases to develop so that it may be more appropriate to examine mortality experience only after a cer- $\tilde{\sigma}$ tain lag period has elapsed. Table 7 shows the $N$ observed deaths and SMRs by cause for the total $N$ cohort by latency since hire. In general, persons fol- $\omega$ lowed up for less than 10 years from hire date had exceptionally low SMRs. Among those followed up for 10-19 years, the only significant SMR was for $\stackrel{D}{\Phi}$ suicide $(S M R=148.7, \mathrm{p}<0.05)$. In the same group ?SMRs for cancer of the rectum (131.8), lymphosarcoma and reticulosarcoma $(180 \cdot 7)$, other lym- $\stackrel{\odot}{\Phi}$ phatic tissue cancers (263.3), and emphysema (145.3) 
were raised, but none was significant. For those followed up for more than 20 years, no significant excess from any cause was found. In fact, most causes of death were either similar to or less than expected (significant or otherwise).

No obvious upward trend by latency was found, except for cancer of the digestive system. Nevertheless, when the subcategories within the digestive system were examined, no consistent trend was evident.

\section{ANALYSIS BY DURATION OF EMPLOYMENT}

Table 8 gives the observed number of deaths and SMRs, by cause, for the total cohort by duration of employment at the refineries. There was a slight downward trend of overall mortality, but the magnitude of the trend was small. More prominent downward gradients were observed for lung cancer, kidney cancer, and cirrhosis of the liver.

The only obvious upward trends were those for all lymphatic and haematopoietic cancer and for suicide. For suicide, however, the magnitude of the trend was small. When the subcategories of lymphatic and haematopoietic cancer were examined, lymphoma and reticulosarcoma, Hodgkin's disease, and leukaemia showed a slight upward trend, albeit no death was observed for any of these three causes in the shortest duration of employment ( $<5$ years) group. On the other hand, there was a downward trend for other lymphatic tissue cancers.

\section{ANALYSIS BY HIRE DATE}

The refinery workers in the study were potentially exposed to benzene, which has been implicated in several previous studies for increasing the risk of leukaemias and lymphomas. ${ }^{1314}$ Occupational standards for benzene have undergone several changes historically. In 1947 the recommended benzene standard (8-hour TWA) was lowered from $100 \mathrm{ppm}$ to $50 \mathrm{ppm}$, and was further reduced to $35 \mathrm{ppm}$ in 1948 .

In this analysis the cohort was divided into those hired in or before 1948, and those hired after 1948, in order to assess mortality risks before and after this reduction. The most striking contrast was in the SMRs for lymphatic and haematopoietic cancer before 1948 (120.9 based on 58 observed deaths) and after 1948 (46.2 based on 6 observed deaths). For lymphosarcoma and reticulosarcoma, the SMRs were 149.6 (16 observed deaths) and 36.6 (1 observed death), respectively. All 22 deaths from leukaemia $(\mathrm{SMR}=110 \cdot 2)$ occurred among those hired in or before 1948, and no deaths from leukaemia were observed among those hired after 1948. Similarly, all six deaths $(\mathrm{SMR}=103.6)$ from diseases of the blood occurred among those hired in or before 1948 and none among those hired after 1948.
Similar analyses by hire date were performed separately for the refineries. The contrast in total lymphatic and haematopoietic cancer mortality and its subcategories by hire date appeared to come primarily from Richmond. In fact, for those hired in or before 1948 at the Richmond refinery, the SMR for lymphosarcoma and reticulosarcoma was 186.9 (13 observed deaths, $p<0.05$ ) and that for other lympathic tissue cancer was 185.8 (14 observed deaths, $p<0.05$ ). For these two categories, only one death (from other lymphatic tissue cancer) occurred among employees hired after 1948.

When comparing mortality by hire date, one must remember that hire date is confounded by latency. In this analysis, however, those hired after 1948 could have a maximum latency of 32 years (1948-80), which appeared to be reasonably adequate.

\section{ANALYSIS FOR LYMPHATIC AND}

HAEMATOPOIETIC CANCER

Based on results from previous studies, lymphatic and haematopoietic cancer was an area of concern in this study of refinery workers and so this cause of death, as well as the subcategory, leukaemia, was examined in greater detail. Table 9 shows the mortality from all lymphopoietic cancers, which includes all lymphatic cancer and leukaemias, both by latency and duration of employment. Although total lymphopoietic cancer had an SMR of only 105.0 (not significant), analysis by duration of employment and latency (time since first employment) shows some upward trend in SMRs by both variables, albeit with small numbers in the short term categories. For leukaemia, a subgroup of the lymphopoietic category, the data show a somewhat similar trend but with even smaller numbers in the short term categories for either latency or duration, and none of the SMRs was significant.

As mentioned earlier, the excess in lymphopoietic cancer mortality was entirely due to deaths from nonHodgkin's lymphatic cancer. In an attempt to identify any common work assignments and to assess the

Table 9 Observed deaths and SMRs, by latency and duration of employment from lymphatic and haematopoietic cancer among Richmond and El Segundo refinery workers

\begin{tabular}{|c|c|c|c|c|c|c|c|}
\hline \multirow[t]{3}{*}{ Latency } & \multicolumn{7}{|c|}{ Duration of employment (years) } \\
\hline & \multicolumn{2}{|l|}{$<5$} & \multicolumn{2}{|c|}{$5-14$} & \multicolumn{2}{|c|}{$\geqslant 15$} & Total \\
\hline & $\overline{O b s}$ & $S M R$ & $\overline{O b s}$ & $S M R$ & $\overline{O b s}$ & $S M R \overline{O b s}$ & $S M R$ \\
\hline $\begin{array}{l}<10 \\
10-19 \\
\geqslant 20 \\
\text { Total }\end{array}$ & $\begin{array}{l}0 \\
2 \\
1 \\
3\end{array}$ & $\begin{array}{l}\overline{94 \cdot 7} \\
44 \cdot 2 \\
38 \cdot 0\end{array}$ & $\begin{array}{r}2 \\
5 \\
4 \\
11\end{array}$ & $\begin{array}{l}65 \cdot 3 \\
89 \cdot 4 \\
74 \cdot 2 \\
78 \cdot 3\end{array}$ & $\begin{array}{r}0 \\
5 \\
45 \\
50\end{array}$ & $\begin{array}{ll}- & 2 \\
143.8 & 12 \\
126.6 & 50 \\
128.1 & 64\end{array}$ & $\begin{array}{r}30 \cdot 4 \\
107 \cdot 3 \\
115 \cdot 7 \\
105.0\end{array}$ \\
\hline
\end{tabular}

Mean exposure $=25.9$ years.

Mean latency $=32.9$ years. 
availability of specific exposure information among these deaths a thorough review of the complete work histories of the 37 deaths from non-Hodgkin's lymphatic cancer (ICD 200, 202, 203, 208) was undertaken. The review was conducted by members of the medical, industrial hygiene, and safety departments, who were knowledgeable about both past and current operations and job task at the refineries.

The figure shows the job locations of the 37 deaths by year of hire, year of death, and ICD code. The figure was developed to facilitate the identification of common work locations by specific ICD category and calendar time. By visualising or drawing a line through a single year or band of time (a five year $\overrightarrow{\vec{P}}$ period across the graph for example), it is easier to $\frac{3}{3}$ identify any clusters by work locations. No obvious $\supset$ similarity in job assignments was evident from this figure. At first glance the number of maintenance jobs appeared somewhat high in proportion to other work locations; 22 of the $37(59 \%)$ had ever worked in maintenance at some time during their employment at 3 the plant. Excluding cases 9 and 33, who worked for $\dot{\gamma}$ less than one year in maintenance, the percentage $\frac{\circ}{3}$ becomes $54 \%$ (20/37). At Richmond this percentage is $50 \%(14 / 28)$. These percentages were not unusual because typically close to half the refinery workforce

Table 10 Observed deaths, expected deaths, and SMRs for malignant cancer of the brain and CNS (191-192), benign brain tumours (225.0), and brain tumours, nature unspecified (238) for total cohort at the Richmond and El Segundo refineries

\begin{tabular}{|c|c|c|c|c|}
\hline Cause of death (8th ICD) & Observed deaths & Expected deaths & $S M R$ & $95 \%$ Confidence interval \\
\hline \multirow{2}{*}{$\begin{array}{l}\text { Malignant cancer of brain and } \\
\text { CNS (191-192) } \\
\text { Benign brain tumours }(225 \cdot 0) \\
\text { Brain tumours, nature unspecified (238) } \\
\text { Malignant, benign, and unspecified brain } \\
\text { tumours }(191-192,225 \cdot 0,238)\end{array}$} & $\begin{array}{r}22 \\
0 \\
1\end{array}$ & $\begin{array}{r}17.48 \\
1.16 \\
4.36\end{array}$ & $\begin{array}{c}125 \cdot 9 \\
0 \\
22 \cdot 9\end{array}$ & $\begin{array}{l}78 \cdot 7-190 \cdot 7 \\
0 \cdot 6-127 \cdot 2\end{array}$ \\
\hline & 23 & 23.00 & $100 \cdot 0$ & $65 \cdot 4-150 \cdot 2$ \\
\hline
\end{tabular}

Expected deaths from benign brain tumours (225.0) and brain tumours, nature unspecified (238), were based on 1968 United States death rates obtained from the National Center for Health Statistics. 
is concerned in maintenance activities at any one time. Further examination of specific crafts among the cases who worked in maintenance showed no unusual clustering. The most commonly listed craft was that of "still" or "tank" cleaner, but only five individuals had this job title. Furthermore, three of the five held this job for three years or less. The lack of adequate historical exposure data did not permit further analysis by quantification or even qualification of exposure to specific chemical agents.

ANALYSIS FOR MALIGNANT, BENIGN, AND "NATURE UNSPECIFIED" BRAIN TUMOURS The petrochemical industry has had considerable interest in brain tumours and, specifically, brain cancer, since the publication of several studies that reported a raised risk. ${ }^{7915-17}$ Frequently, brain cancers are incompletely certified and lack histological diagnosis. To overcome this underreporting of malignant brain tumours, and to recognise the lethality of benign brain tumours, some investigators (NIOSH, for example) have combined benign and nature unspecified brain tumours with brain cancer as a category straddling three ICD divisions.

Table 10 shows the analysis of all brain tumours (classified as malignant, benign, or unspecified) among men. National age-race-sex-specific death rates for benign brain tumour (8th ICD 225.0) are not published but were obtained for this analysis by special request to the National Center for Health Statistics (NCHS). In calculating the expected deaths for benign brain tumours the 1968 United States death rates were used. Before 1968, no separate rates were generated for benign brain tumours. In addition, SMRs for brain tumours, nature unspecified (8th ICD 239) are also presented in the table. Although there was a slight non-significant excess in malignant cancer of the brain and central nervous system, deficits were observed for benign and unspecified brain tumours. When all three categories were combined, the observed number of deaths (23) was identical with the expected (23.0).

\section{Discussion}

The total cohort was relatively large and was adequate for examining mortality from several individual causes. Although the Richmond and El Segundo refineries had a few differences in cause specific mortality, their experience was sufficiently similar to justify combining them in the analysis in order to increase statistical power (in analysing less common causes of death).

The all causes SMR was only $72 \cdot 4$, a highly significant reduction from that expected on the basis of United States statistics, and somewhat lower than that often found in cohorts demonstrating the "healthy worker effect." Comparison of the United States and the local county cancer statistics indicates that similar results would have been obtained had we compared our cohort against county rates. We can see no methodological flaw that might have produced this low SMR; we therefore conclude that this cohort has an unusually low mortality rate. Presumably, this is a combination of the usual healthy worker effect (selection of the healthy for employment), a relative absence of serious risk from the job, and the effects of continuing employment with its several benefits (including health care), and lifestyle.

Because of the essentially negative findings, we calculated the minimum relative risks that were detectable with $80 \%$ power at the 0.05 level for several causes of death of interest. For most malignant causes of death that have been implicated in previous studies of refinery workers, the cohort as a whole was sufficiently large to detect with $80 \%$ power any significant excess risk greater than twofold. In particular, the minimum relative risk was 2.03 for brain cancer, 1.47 for respiratory cancer, 1.70 for stomach cancer, 1.84 for leukaemia, 1.79 for nonHodgkin's lymphomas, and $2 \cdot 14$ for kidney cancer.

The petroleum industry, especially in Texas, has had considerable interest in brain tumours and brain cancer. To overcome the frequent underreporting of malignant brain tumours, and to recognise the lethality of benign brain tumours, we have combined benign and "nature unspecified" brain tumours with brain cancer as a category straddling three ICD divisions (table 10). Our analysis indicates that there was a slight (non-significant) excess in malignant brain cancer (primarily from Richmond) but a deficit in the other two categories, particularly in the category of brain tumours, nature unspecified. For the three categories as a whole, the observed mortality was exactly as expected. One possible explanation for this observation is that diagnostic practice and death certification for brain cancer in Richmond and El Segundo, both within large metropolitan areas (San Francisco and Los Angeles, respectively), are more specific and complete than the nation as a whole (19 of the 22 subjects with malignant brain cancer lived in the San Francisco Bay area or Los Angeles County at the time to their deaths). A previous study had indicated that among the medically well insured, a high level of investigation may lead to an increased frequency of the diagnosis of brain cancer, giving an apparent (not real) increase in reported incidence rates. ${ }^{18}$ Within the Chevron cohort, however, other explanations cannot be ruled out.

For malignancies of the lymphopoietic (lymphatic and haematopoietic) system, there was a slight, nonsignifiant excess (the study had $80 \%$ power to exclude 
only an SMR of 150 or higher). Furthermore, there was a trend of increasing SMRs with both increasing duration of employment and latency since first employment. This pattern was compatible with an exposure related disease, although numbers were too small (within each time interval) to be conclusive of a trend.

When the subcategories within all lymphopoietic cancer were examined, the excess was found to come entirely from lymphosarcoma and reticulosarcoma, as well as other lymphatic tissue cancers (nonHodgkin's lymphoma, multiple myeloma, and polycythaemia vera).There was a deficit in Hodgkin's disease (SMR $=52 \cdot 1$, not significant), but the number was small. For leukaemia, there was also a slight deficit (SMR $=88 \cdot 3$, not significant).

As previously noted, Richmond and El Segundo refineries differed somewhat, with Richmond having a significant excess in other lymphatic cancer for those hired before 1948. Although leukaemia had a raised SMR for both refineries among pre-1949 employees, no cases occurred at either refinery in those workers hired after 1948.

Between refineries, there was some difference in total lymphopoietic cancer experience, with Richmond showing an excess (SMR $=118$, not significant) and El Segundo a deficit (SMR $=82$, not significant). The difference between the two refineries was not significant.

Among the work location subcohorts, there were no major differences in mortality patterns. Maintenance workers had less of an excess of lymphopoietic cancer than did laboratory or operating personnel. The latter was the only cohort with an excess of leukaemia.

We suggest caution in interpreting mortality patterns, by diagnosis, within the category of lymphatic and haematopoietic cancer (ICD 200-209). With the possible exception of Hodgkin's disease of recent diagnosis, there is considerable opportunity for misdiagnosis, misclassification, and even multiple diagnoses among the other rubrics in the category. For instance, both lymphosarcomas and myeloproliferative syndromes often end in acute myelocytic leukaemias. Furthermore, it has been suggested that most lymphatic and haematopoietic malignancy reflects an oncogenic insult to the precursor stem cell series. ${ }^{1920}$

Because only first and last job were coded, it is difficult to draw firm conclusions concerning the effect (if any) of subcohort membership on a particular disease category of interest, such as the lymphatic and haematopoietic cancer. The split into subcohorts is most useful in examining general patterns only. In view of the excess in mortality from non-Hodgkin's lymphatic cancer a review of the complete work his- tories of the 37 deaths from this cause of death was $\frac{3}{8}$

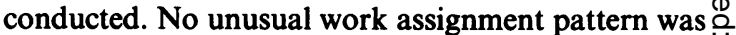
detected and we failed to identify any common $\Rightarrow$ exposure pattern. This observation is consistent with $\stackrel{\mathcal{O}}{+}$ two recent case-control studies of lymphatic cancer $\bar{C}$ among refinery workers ${ }^{21}$ (Divine BJ, unpublished $\frac{\bar{O}}{\bar{N}}$ report). Based on similar methodological strategies, $\frac{\bar{D}}{\vec{D}}$ both concluded that there were no unusual associ- $\stackrel{\square}{2}$ ations by work category (process unit or job task in the case of the study by Divine).

Overall, the study has provided considerable reassurance concerning the long term health of Chevron $\vec{\omega}$

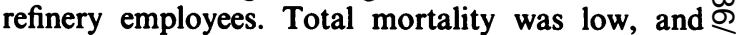
there was no suspicion of an excess risk either for lung $\stackrel{\varnothing}{\Phi}$ cancer (an important issue in both Contra Costa and $?$ Los Angeles counties) or kidney and digestive cancer $\omega$ (previously reported in refinery workers). A recent study suggests that in Contra Costa County, residen- ${ }^{\circ}$ tial exposure to petroleum and chemical air emissions $\stackrel{5}{5}$ was associated with an increased incidence of cancer $\vec{c}$ of the buccal cavity and pharynx. ${ }^{22}$ In our study the SMR for this cancer was significantly low for the total cohort (SMR $=47.0, \mathrm{p}<0.05)$, and for the Richmond refinery in Contra Costa County the SMR was $\vec{\varphi}$ 57.2 (not significant). For cancers of the lympho- $\infty$ poietic system, the study is more equivocal. Although no statistically significant increases in lymphopoietic cancer were observed, the results have raised questions concerning the potential risk to subcohorts or $\overline{0}$ specific exposure categories. While the excess risk $\stackrel{2}{\circ}$ appears confined to non-Hodgkin's lymphatic cancer, $\stackrel{\mathbb{\Omega}}{\Omega}$ not leukaemia, among employees at Richmond hired $\overrightarrow{\vec{O}}$ in or before 1948, and a detailed review of the work 3 histories of these cases did not show any unusual clus- $\bar{T}$ tering of work assignments, we could not rule out the possibility that exposure to a specific chemical or mix- $\bar{O}$ ture caused or contributed to the excess. Without ade- $\frac{\Phi}{3}$ quate historical industrial hygiene data, however, an exposure specific case-control study of lymphatic can- 3 . cer is not possible.

While it is safe to conclude that exposure in general in the refineries, as well as exposures to many specific 을 chemical agents, have decreased sizeably since the 1930s to mid-1950s (the time when most cases were employed), it will be necessary to follow up the full cohort for an additional period to assess adequately $\mathcal{N}$ whether the risk of lymphatic cancer has declined in $N$ employees hired after 1950.

Requests for reprints to: Dr O Wong, Environmental ${ }^{O}$ Health Associates, Inc, 520 Third Street - Suite 208, Oakland, CA 94607.

\section{References}

${ }^{1}$ Hanis NM, Stavraky KM, Fowler JL. Cancer mortality in oil $\frac{\text { }}{\mathbb{Q}}$ 
refinery workers. JOccup Med 1979;21:167-74.

${ }^{2}$ Hanis NM, Holmes TM, Shallenberger LG, Jones KE. Epidemiologic study of refinery and chemical plant workers. JOccup Med 1982;24:203-12.

${ }^{3}$ Rushton L, Alderson MR. An epidemiological survey of eight oil refineries in Britain. Br J Ind Med 1981;38:225-34.

${ }^{4}$ Rushton L, Alderson MR. A case-control study to investigate the association between exposure to benzene and deaths from leukemia in oil refinery workers. Br J Cancer 1981;43:77-84.

${ }^{5}$ Schottenfeld D, Warshauer ME, Zauber AG, Meikle JG, Hart BR. A prospective study of morbidity and mortality in petroleum industry employees in the United States - a preliminary report. In: Banbury report No 9. Quantification of occupational cancer. New York: Cold Spring Harbor Laboratory, 1981:247-65.

${ }^{6}$ Tabershaw/Cooper Associates. A mortality study of petroleum refinery workers 1974. (American Petroleum Institute Medical Research Report No EA 7402.)

${ }^{7}$ Thomas TL, Waxweiler RJ, Moure-Eraso R, Itaya S, Fraumeni JF. Mortality patterns among workers in three Texas oil refineries. JOccup Med 1982;24:135-41.

${ }^{8}$ Tsai SP, Wen CP, Weiss NS, Wong O, McClellan WA, Gibson RL. Retrospective mortality and medical surveillance studies of workers in benzene areas of refineries. J Occup Med 1983; 25:685-92.

${ }^{9}$ Wen CP, Tsai SP, Weiss NS, McClellan WA, Gibson RL. A population-based cohort study of brain tumour mortality among oil refinery workers with a discussion of methodological issues of SMR and PMR. In: Banbury report No 9. Quantification of occupational cancer. New York: Cold Spring Habour Laboratory, 1981:413-32.

${ }^{10}$ Wen CP, Tsai SP, McClellan WA, Gibson RL. Long-term mortality study of oil refinery workers. I. Mortality of hourly and salaried workers. Am J Epidemiol 1983;118:526-42.

${ }^{11}$ World Health Organisation. Manual of the international statistical classification of diseases, injuries and causes of death, based on the recommendations of the eighth revision conference, 1965, and adopted by the nineteenth World Health Assembly. Vols I and II. Geneva: WHO, 1967, 1968.

12 Marsh GM, Preininger M. OCMAP: a user-oriented occupational cohort mortality analysis program. American Statistician 1980;34:254.

${ }^{13}$ Rinsky RA, Young RJ, Smith AB. Leukemia in benzene workers. Am J Ind Med 1981;2:217-45.

${ }^{14}$ Wong $O$. An industry-wide mortality study of chemical workers occupationally exposed to benzene. (Technical report submitted to Chemical Manufacturers Association, 1983.)

${ }^{15}$ Theriault G, Goulet L. A mortality study of oil refinery workers. JOccup Med 1979;21:367-70.

${ }^{16}$ Alexander V, Leffingwell SS, Lloyd JW, et al. Brain cancer in petrochemical workers: a case-series report. Am J Ind Med 1980;1:115-23.

17 Waxweiler RJ, Alexander V, Leffingwell SS, et al. Mortality from brain tumor and other causes in a cohort study of petrochemical workers. JNCI 1983;70:75-81.

${ }^{18}$ Greenwald P, Friedlander BR, Lawrence CE, Hearne T, Earle K. Diagnostic sensitivity bias - an epidemiologic explanation for an apparent brain tumor excess. JOccup Med 1981;23:690-4.

${ }^{19}$ Bakhshi A, Minowada J, Arnold A, et al. Lymphoid blast crises of chronic myelogenous leukemia represent stages in the development of B-Cell precursors. N Engl J Med 1983;309:826-31.

${ }^{20}$ Kersey JH. Chronic myelocytic (multipotent-stem-cell) leukemia. N Engl J Med 1983;309:851-2.

${ }^{21}$ Thomas TL, Waxweiler RJ, Crandall MS, White DW, MoureEraso R, Fraumeni JF. Cancer mortality patterns by work category in three Texas oil refineries. Am J Ind Med 1984;6:3-16.

${ }^{22}$ Kaldor J, Hanis JA, Glazer E, et al. Statistical association between cancer incidence and major cause of mortality, and estimated residential exposure to air emissions from petroleum and chemical plants. Environ Health Persp 1984;54:319-32. 